\title{
EMA's parallel advice workshop bridges regulatory and reimbursement divide
}

Regulators and health technology assessment bodies discussed early experiences with pilot programmes in which they work together to provide drug developers with joint advice on the design of clinical trials.

\section{Suzanne Elvidge}

There's more to successfully marketing a drug than just getting approval. Achieving reimbursement by satisfying the requirements of increasingly cost-conscious payers - in particular those receiving input from health technology assessment (HTA) bodies in Europe - can be a difficult barrier to overcome. In the latest move to smooth the way and coordinate the data requirements of regulatory and HTA bodies, the European Medicines Agency (EMA) held a 280-person workshop in London, UK, in late November to discuss the provision of parallel scientific advice.

"This is the first workshop where we have tried to bridge these two worlds together to share views," said Guido Rasi, Executive Director of the EMA, in a press statement.

The need for a joined up approach is clear. Long, large and expensive clinical trial programmes for investigational drugs are designed primarily to answer regulators' questions about drug safety and efficacy. But growing pressure on health-care budgets, owing to spending cuts and changing population demographics, means that HTAs cannot support reimbursement of all expensive new drugs, especially if the extent of their benefit in the real world is not yet well defined. And the current approach to clinical trials, despite their high costs, often provides insufficient insight into the real-world efficacy or comparative cost effectiveness that HTAs need to make their decisions.

If regulators and HTAs could provide their scientific advice together, drug companies could reshape clinical programmes accordingly, improving the odds of developing a reimbursable drug and producing data that are more useful to all stakeholders. Although presentations at the EMA's workshop showed that this is easier said than done, drug developers, regulators, payers, HTAs and patients are at least on the same page. "I was surprised to hear the same from all sides - that this is a necessary process and should be streamlined and continued," says Jan Mueller-Berghaus, a regulator at Germany's Paul-Ehrlich-Institut, who presented at the conference. "I actually expected to see more resistance."

Paolo Morgese, director at the Deerfield Institute, an investment group, was also encouraged. "I have been working at joint European HTA initiatives since 2005 and I have seen progress over the years, despite different views among stakeholders. This workshop has given me the impression that there is now a broad consensus on the importance of joint EMA-HTA advice activities."

\section{Piloting parallel advice}

To move from consensus to practice, the EMA and EUnetHTA - a network of HTAs across Europe - began working together in 2010 to align regulatory benefit-risk assessments with HTA evaluations. Under this partnership, the EMA launched a pilot project to start testing a parallel advice process.

Companies approach the regulator early on, with initial informal discussions specifying which HTAs to involve and checking the scope and clarity of the sponsor's questions. Face-to-face meetings with the EMA and HTAs are followed within 70 days by official advice from the involved parties. Around 25 parallel advice procedures have been completed or are ongoing, for a wide range of new innovative products. Six more are expected to start in 2014 , and the first drugs could graduate from the programme within 3-5 years.

An early analysis of 18 of the pilots, presented by Mueller-Berghaus at the workshop, showed that nine procedures were carried out at Phase I and seven at Phase II; over half involved three or four HTAs; and the most common questions from sponsors were about study design and end points. He also found that regulators and HTAs tended to ask for different end points, and that HTAs seemed less concerned with methodology and statistical considerations.

One outcome of the pilot, says MuellerBerghaus, is that it allowed HTAs and regulators to see each other as equal partners and to start to identify the critical divergences. "However because the parallel process requires extra resources and preparation, the HTAs and national agencies will have to convince their respective organizations that it is worthwhile to invest," he cautions.

The early results of a separate pilot programme headed up by the EUnetHTA, in which multiple HTAs gave advice to sponsors while the EMA acted as an observer, offered some additional lessons. Mira Pavlovic and François Meyer, of the French HTA Haute Autorité de Santé, reported on ten such pilot processes and concluded that these are best carried out before Phase III, and sometimes even before Phase II. They also suggested that the best route to efficient and effective meetings was to focus on one indication at a time, and to have a minimum of five HTAs but not more than ten involved.

"The number of agencies at the meeting matters," explains the UK National Institute for Health and Care Excellence (NICE)'s senior scientific adviser Leeza Osipenko, who also presented at the meeting and has been involved in a number of parallel advice pilots. "If too many agencies are invited, then it's hard to have meaningful discussions." The number of questions asked by sponsors matters too. Encouragingly, she adds, "so far we have found more commonalities than differences in the views expressed by different parties involved in parallel advice."

From industry's standpoint, a European Federation of Pharmaceutical Industries and Associations (EFPIA) industry survey showed that the drug developers are nevertheless still worried about the lack of consensus among different HTAs, as well as the time and resources needed and the lack of clear procedures.

As a next step, which may address at least industry's procedural concerns, the EMA is now developing parallel advice guidance that will detail the timelines and actions through which applicants can seek simultaneous feedback. Draft guidance will be published for public consultation in early 2014. This draft is part of the joint 3-year work plan from the EMA and EUnetHTA, which also calls for the development of better approaches for collecting post-authorization data that can inform both regulators and HTAs.

The Shaping European Early Dialogues for health technologies (SEED) consortium, which consists of 14 national and regional HTA bodies and was financed by the European Commission and launched in October 2013, is also exploring how to best conduct early dialogues between stakeholders within the context of a parallel advice setting. 